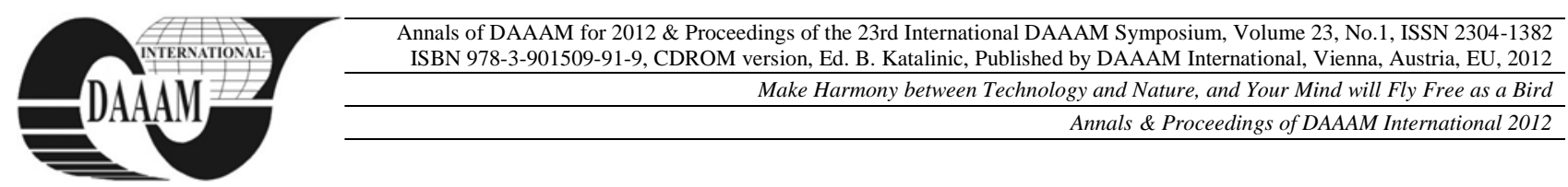

\title{
MODEL PERFORMANCE APPRAISAL IN FORESTRY UNITS
}

\author{
CHERECHES, C[atalin]; TOADER, R[ita]; RADULESCU, C[orina] M[ichaella]; \\ ZIMA, L[iliana] A[dela] \& SABOU, S[imona]
}

\begin{abstract}
This paper presents some aspects of the performance assessment objectives, contents of performance appraisal, calculation of individual performance according and a mathematical model of performance appraisal in units of forestry.

Keywords: models, performance, assessment, appraisal, human resources, methods
\end{abstract}

\section{INTRODUCTION}

Studies by experts in the field, mainly human performance assessment have, in time developing techniques and assessment methods, which are in a permanent dynamic and have eliminated some errors in measuring.

Performance management focuses on ways to motivate employees to improve their performance. The goal of the performance management process is performance improvement, initially at the level of the individual employee, and ultimately at the level of the organization.

Leadership is an essential variable leads to an improved ability to lead and organizational performance. describes the hypothetical relationship between leadership and organizational performance. With time a certain organizational culture, how leadership is exercised largely determines the capacity of management. But increased management capacity does not necessarily lead to higher organizational performance of forestry.

Performance evaluation is a necessary and beneficial process, which provides annual feedback to staff members about job effectiveness in units of forestry.

A correct assessment and using efficient performance assessment methods can improve the accuracy of the data according to which employees are lead, and predictions are made in order to ensure human resources.

The performance assessment systems constitute an intrinsic and special part within the management system in general and the human resources management system in particular, the latter circumscribing to a certain extent to the organizational environment.

Performance appraisal feedback research suggests that agreement of others' performance feedback with one's own views strongly determines feedback reactions, yet inconsistent results of feedback interventions motivate a search for additional influences.

Performance appraisal allows a variety of benefits:
- Provides structured opportunity for staff at different levels;

- Provide a forum for individual feedback on work performance;

- Staff can present their views and ideas;

- Allows face to face, one-to-one discussion;

- Clarifies what is expected of an individual and how this relates to the objectives of forestry;

- Chance to explain to staff about developments affecting the ward / department / team;

- Opportunity to thank staff for a job well done;

- Opportunity for you to influence attitudes and behaviours.

\section{CONTENTS OF PERFORMANCE APPRAISAL}

1. Quality of work- ability to meet standards of quality, use of time and volume of work accomplished, work output matches the expectations established.

2. Quantity of work-competence and efficiency of work regardless of volume.

3. Teamwork-establish and maintain effective working relationship with others, follows instructions of supervisor, contributing work and effort to group performance to meet agreed upon objectives and achieve team success.

4. Initiative- recommends and creates own procedures, develop and implement new methods, solutions, accepts additional challenges and responsibilities.

5. Interpersonal relations-the extent to which the employee is cooperative, considerate, and tactful in dealing with supervisors and subordinates.

6. Communications abilities- ability to listen and understand information, demonstrates respect for all individuals in all forms of communication, regardless of their background or culture.

7. Planning and organizing -adapting to changes and using resources effectively, Setting objectives, establishing priorities, developing plans, prioritizing work to meet deadlines.

8. Problem analysis and decision making-anticipating problems and facilitate problem resolution, understanding practical and workable solutions, making decisions and providing information.

Performance appraisal methods are: 
1. Critical incident method

2. Weighted checklist method

3. Paired comparison analysis

4. Graphic rating scales

5. Essay Evaluation method

6. Behaviorally anchored rating scales

7. Performance ranking method

8. Management By Objectives (MBO) method

9. 360 degree performance appraisal

10. Forced ranking (forced distribution)

11. Behavioral Observation Scales.

\section{THE ANALYSIS OF MOTIVATION FACTORS IN UNITS OF FORESTRY}

The questionnaire is meant the situation precisely the topic of social landmarks. It will evolve as the degree of integration in group work, attitude towards work, operating conditions, level of aspiration, satisfaction, organizational dysfunctions in space of Forestry Department of Maramureş county.

Given the size of the population studied (126 employees of the Forestry Department of Maramureş county), we opted to perform the research on a representative sample consisting of 25 subjects, representing $19 \%$ of the population studied. Data collection and interpretation of results were used 18 factors whose definitions are listed below. Each factor has the general perception of subjects concerning aspect of the analysis:

1. A. Professional achievement;

2.Recognition for achievement;

3. Work itself;

4. Responsibility;

5. Advancement in the hierarchy;

6. Personal and professional development

7. Shared values

8. Organizational policies and procedures;

9. Supervision;

10.Environment;

11.Remuneration;

12. Personal life;

13. Relations with colleagues;

14. Relations with other departments;

15. Perceived status;

16. Stress;

17. Quality products and services;

18. General satisfaction.

In the work itself, there is a surfeit of high-level employees are not satisfied with the nature of their work $28 \%$ dissatisfied and $28 \%$ partially satisfied. More than one third of the employees consider work being interesting $32 \%$ satisfied and $12 \%$ consider challenging.

Most employees, $36 \%$ are partially satisfied with the responsibilities involved in job positions, while the number dissatisfied is $24 \%$, implying an overall negative image $60 \%$. The results obtained in this factor shows that managers are not concerned at a high level of quality responsibilities they hold employees and, especially, of how employees can fulfill these responsibilities. Working methods such as the freedom to make decisions, identify ways to improve processes and ability to manage their own budgets apparently not used at high levels by managers.

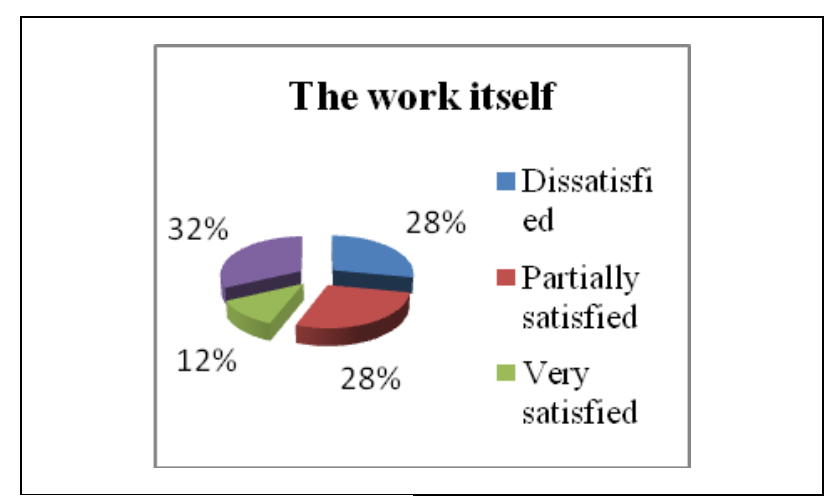

Fig. 1. Responses to the work itself

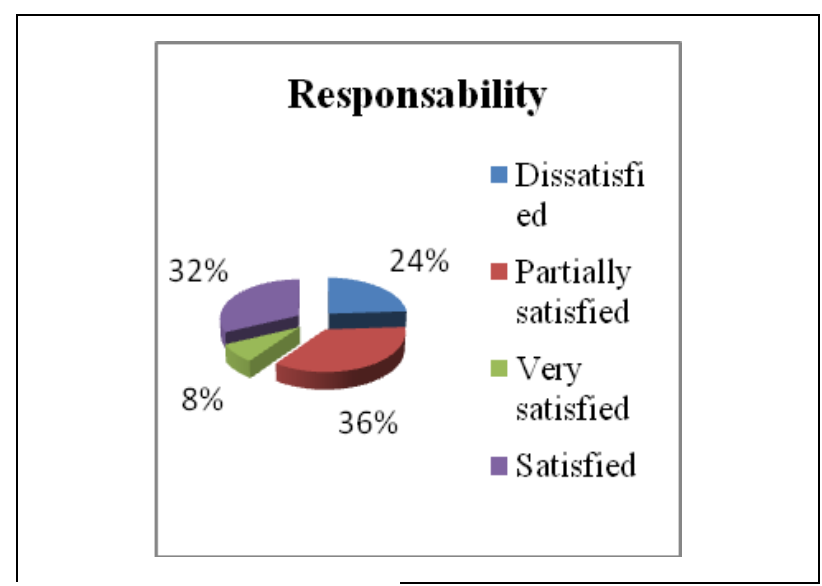

Fig. 2. Responses to responsibility

Professional development at work.

Most employees are partially satisfied - $40 \%$ dissatisfied - $32 \%$ of their professional development at work, development is primarily related to performing the service.

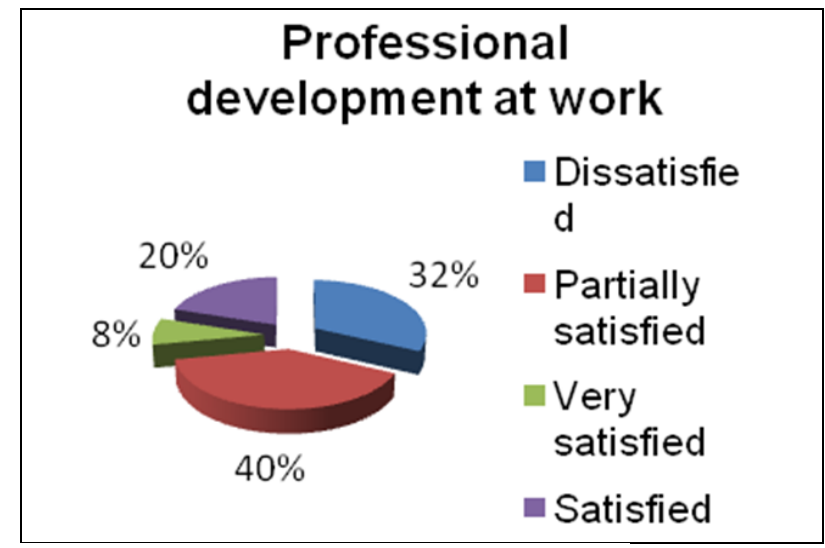

Fig. 3. Responses to professional development at work

In relationships with colleagues, $48 \%$ said very satisfied and $36 \%$ satisfied with these relationships good and means well with teammates. The proportion is similar in relations with other departments $32 \%$ very satisfied and $24 \%$ satisfied. 


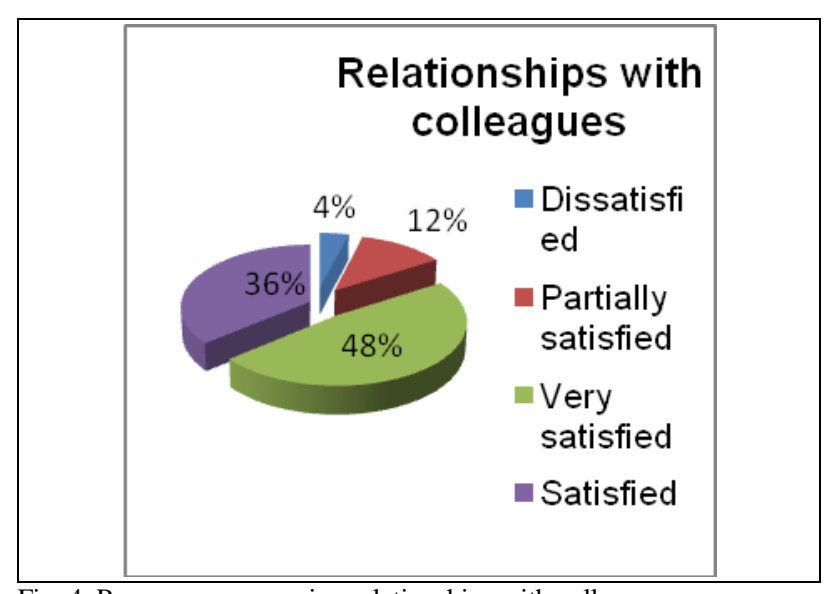

Fig. 4. Responses concerning relationships with colleagues

\section{MODELLING PERFORMANCE APPRAISAL IN UNITS OF FORESTRY}

Looking at ways by which the performance appraisal of human resources in forestry units are found practically the only formal instrument used in performance appraisal at the individual and within a year, is the file that meet certain characteristics evaluation of technology performance through assessment scales.

Using a mathematical model based on the degree of importance of each evaluation criteria and scores given to improve the human performance evaluation of forestry units eliminating some errors in the evaluation.

In the assessment and quantify by how to make attribute evaluator by a particular employee, will take into account not only the quality of their achievement, but also the degree of importance of criteria for assessing human performance.

Were applied in 30 units of forestry human resources performance evaluation questionnaires.

Results of the questionnaires have emphasized that the evaluation methods currently used are not effective, do not take into account the degree of importance of performance evaluation criteria for human resources.

Analyzing responses to questionnaires managers in the 30 units resulted forestry need to introduce a new model of human performance evaluation that takes into account the degree of importance of evaluation criteria.

A correct assessment system has to meet the following conditions:

- Careful preparation and dissemination of the value system and performance assessment procedures with the aim of preventing adverse reactions or challenging the results;

- The existence of a formal assessment tool;

- Using criteria that limit the absolute power of management;

- Personally knowing the assessed person and permanent contact with them;

- Continuous training for managers regarding the assessment activity;

- The existence of a revising system for incorrect assessments, done by superiors;

- Counselling and support for those with poor performances in order to offer them the possibility to improve.

Scales as follows for this performance appraisal form:

1. Unsatisfactory: major improvements needed;

2. Needs Improvement: less than Satisfactory, could be doing better;

3. Meets Expectations: performing duties as directed with minimal supervision

4. Excellent: performing all duties in a cost-effective manner with positive, measurable results

5. Outstanding: performing at a level above and beyond the duties of the current position's requirements.

The evaluation criteria of individual performance are the following:

- The level of meeting the performance standards;

- Assume responsibility;

- Meet the task work;

- Initiative and creativity.

In developing the model of human performance evaluation should be considered in determining variability describing performance and assigning scores, the performance standards.

Every employee is a special case, being considered independently and not reported to the group or team to which it belongs.

General equation:

$$
\begin{gathered}
P=f\left(c_{1}, c_{2}, c_{3}, c_{4}\right) \\
P=b_{0}+b_{1} c_{1}+b_{2} c_{2}+b_{3} c_{3}+b_{4} c_{4}+b_{1234} c_{1} c_{2} c_{3} c_{4}
\end{gathered}
$$

$$
b_{0}=45,5 \quad b_{1}=-0,5 \quad b_{2}=-1,25 \quad b_{3}=-1,5 \quad b_{4}=-0,75
$$

\begin{tabular}{|l|c|c|c|c|}
\hline Variation level & $\mathrm{x}_{1}(\%)$ & $\mathrm{x}_{2}(\%)$ & $\mathrm{x}_{3}(\%)$ & $\mathrm{x}_{4}(\%)$ \\
\hline Higher level (+1) & 5 & 4 & 5 & 4,5 \\
\hline Basic level (0) & 3 & 3 & 3 & 3 \\
\hline Lower level (-1) & 1 & 2 & 1 & 1,5 \\
\hline Variation level $\left(\Delta \mathrm{x}_{\mathrm{i}}\right)$ & 2 & 1 & 2 & 1,5 \\
\hline
\end{tabular}

Tab. 1. Variation of level

The potential problems that need consideration in approaching performance assessment as a component of the performance management system are the following:

- The necessity of creating a performance culture;

- Involving managers at higher levels in the assessment process in order to satisfy both individual and organisational needs;

- The influence of performance management upon the role of its assessment through the appropriate functioning of feedback. 


\begin{tabular}{|c|c|c|c|c|c|}
\hline $\begin{array}{c}\text { Exp. } \\
\text { no. }\end{array}$ & $\mathrm{x}_{0}$ & $\mathrm{x}_{1}$ & $\mathrm{x}_{2}$ & $\mathrm{x}_{3}$ & $\mathrm{x}_{4}$ \\
\hline 1 & +1 & +1 & +1 & +1 & +1 \\
\hline 2 & +1 & -1 & +1 & +1 & -1 \\
\hline 3 & +1 & +1 & -1 & +1 & +1 \\
\hline 4 & +1 & -1 & -1 & +1 & -1 \\
\hline 5 & +1 & +1 & +1 & -1 & -1 \\
\hline 6 & +1 & -1 & +1 & -1 & +1 \\
\hline 7 & +1 & +1 & -1 & -1 & +1 \\
\hline 8 & +1 & -1 & -1 & -1 & -1 \\
\hline
\end{tabular}

Tab. 2. Matrix EFC $2^{4}$

\begin{tabular}{|c|c|c|c|c|c|c|}
\hline $\begin{array}{c}\text { Exp. } \\
\text { no. }\end{array}$ & $\mathrm{x}_{1} \mathrm{x}_{2}$ & $\mathrm{x}_{2} \mathrm{x}_{3}$ & $\mathrm{x}_{1} \mathrm{x}_{3}$ & $\mathrm{x}_{1} \mathrm{x}_{4}$ & $\mathrm{x}_{2} \mathrm{x}_{4}$ & $\mathrm{x}_{3} \mathrm{x}_{4}$ \\
\hline 1 & & +1 & +1 & +1 & +1 & +1 \\
\hline 2 & & +1 & -1 & +1 & +1 & -1 \\
\hline 3 & & +1 & +1 & -1 & +1 & +1 \\
\hline 4 & & +1 & -1 & -1 & +1 & -1 \\
\hline 5 & & +1 & +1 & +1 & -1 & -1 \\
\hline 6 & & +1 & -1 & +1 & -1 & +1 \\
\hline 7 & & +1 & +1 & -1 & -1 & +1 \\
\hline 8 & & +1 & -1 & -1 & -1 & -1 \\
\hline
\end{tabular}

Tab. 3. Matrix EFC $\mathrm{x}_{\mathrm{i}} \mathrm{x}_{\mathrm{j}}$

The matrix being orthogonal, coefficients $b_{0}, b_{1}, b_{2}$, $b_{3}, b_{1234}$ are determined with the formula:

$$
b_{\bar{i}}=\frac{\sum_{u=\mathbf{1}}^{N} x_{i_{u}} y_{u}}{\sum_{u=\mathbf{1}}^{N} x_{i_{u}}^{2}} \quad b_{i j}=\frac{\sum_{u=1}^{N} x_{i_{u}} x_{j_{u}} y_{u}}{\sum_{u=\mathbf{1}}^{N}\left(x_{i_{u}} x_{j_{u}}\right)^{2}}
$$

Model performance appraisal in units of forestry

$$
\mathrm{P}=45,5-0,5 \mathrm{x}_{1}-1,25 \mathrm{x}_{2}-1,5 \mathrm{x}_{3}-0,75 \mathrm{x}_{4}-0,05 \mathrm{x}_{1} \mathrm{x}_{2} \mathrm{x}_{3} \mathrm{x}_{4}
$$

\section{Mathematical model verification}

For the values from the higher level:

$$
\begin{aligned}
& \mathrm{X} 1=5 \quad \mathrm{X} 2=4 \quad \mathrm{X} 3=5 \quad \mathrm{X} 4=4,5 \\
& \mathrm{P}=4,6
\end{aligned}
$$

\section{CONCLUSION}

Using a mathematical model based on the degree of importance of each evaluation criteria and scores given to improve the human performance evaluation of the 30 forestry units eliminating some errors in the evaluation.

The model that we have developed as assessment presents the advantage of performance are you regard to the degree of importance of each evaluation criterion.

The model performance appraisal can be successfully implemented in the forestry units

The professional performance evaluation constitutes an undisputable motivational element with regard to the professional activity of every employee.
Performance assessment has a special influence upon the social-economical activity of forestry, the organizational environment in any business organisation.

An important aspect of human resources management is represented by performance assessment within the units of forestry, because through evaluation we can better understand the.

Some of the major appraisal problems in the units of forestry are the subjectivity and the use of inconsistent criteria which may lead to negative attitude toward the appraisal system.

Assessments are influenced by errors systematically placing too much emphasis on individual performance variables and to a lesser extent on the characteristics of current activity, associated with performance.

Important advantages of using in practice the proposed mathematical model are correct assessment among employees, model usability evaluation and elimination of errors.

Of the 30 questionnaires forestry units resulted in a great interest of managers to implement the mathematical model to evaluate human performance, because the model takes into account besides Ede evaluation criteria and their degree of importance.

\section{REFERENCES}

[1] Atanasiu Virginia, (2010) Matematici Aplicate În Economie, Editura Printech, București, pg. 172, ISBN 978-606-521-538-2

[2] Burloiu, P. (1997). Human Resources Management, Published, ISBN 973-663-167-2, LUMINA LEX, Bucharest Certo, C., S. (2001). Modern Management, Published, ISBN 973-95612-4-1, Teora, Bucharest

[3] Cole G., A. (2000). Personnel Management, Published, ISBN: 973-8060-23-0, CODECS, Bucharest

[4] Chişu, A., V. (2002). The Human Resources Specialist's Manual, Published, ISBN 973-20-0597-1, IRECSON, Bucharest

[5] Dedu Silvia, Şerban Florentin, (2009), Applied mathematics in economics, Published, ISBN 978-973-1934-50-1, Editura Teora, Bucharest

[6] Dragoi, Marian, (2008). Forestry Economics and Management, Published, ISBN 978-973-666-286-7, Univerity, Suceava

[7] Ionescu Alexandra (2007). Standards of Performance Criteria and procedures for assessing the performance of human resources, , Published, ISBN: 978-973-749-144-2 , Universitara, Bucharest

[8] Mathis, R. (1999) Human Resources Management, Published, ISBN -13: 978-0538890045, Editura Economică, Economic, Bucharest

[9] Pitariu, H., D. (2000). Human Resources Management Professional Performance Assessment, Published, ISBN 973-751055-0, All Beck, Bucharest

[10] Sterpu, M., Rocşoreanu, C. (2007)- Modeling and simulation of economic processes, Published, ISBN 978-606-510-078-7, Universitaria, Craiova 